\title{
The Compact Neutron Spectrometer at ASDEX Upgrade
}

L. Giacomelli ${ }^{1 *}$, A. Zimbal ${ }^{1}$, K. Tittelmeier ${ }^{1}$, H. Schuhmacher ${ }^{1}$, G. Tardini ${ }^{2}$, R. Neu $^{2}$ and the ASDEX Upgrade Team ${ }^{* *}$

1 Physikalisch-Technische Bundesanstalt (PTB), Bundesallee 100, 38116 Braunschweig, Germany

2 Max Planck Institut für Plasmaphysik, EURATOM Association, Boltzmannstrasse 2, 85748 Garching, Germany

* now at CNISM, Dipartimento di Fisica, Università degli Studi di Milano-Bicocca, and Istituto di Fisica del Plasma, Associazione EURATOM-ENEA-CNR, Milano, Italy

** see the Appendix of A. Kallenbach et al., "Overview of ASDEX Upgrade Results", Proc. of the $23^{\text {rd }}$ IAEA Fusion Energy Conference, 11-16 October 2010, Daejeon, Rep. of Korea, Vol. IAEA-CN-180 (2010), OV/3-1

\begin{abstract}
The first neutron spectrometer of ASDEX Upgrade (AUG) was installed in November 2008. It is a compact neutron spectrometer (CNS) based on a BC501A liquid scintillating detector, which can simultaneously measure $2.45-\mathrm{MeV}$ and $14-\mathrm{MeV}$ neutrons emitted from deuterium (D) plasmas and $\gamma$ radiation. The scintillating detector is coupled to a digital pulse shape discrimination data acquisition (DPSD) system capable of count rates up to $10^{6} \mathrm{~s}^{-1}$. The DPSD system can operate in acquisition and processing mode. With the latter $n-\gamma$ discrimination is performed off-line based on the two-gate method. The paper describes the tests of the CNS and its installation at AUG. The neutron emission from the D plasma measured during a discharge with high auxiliary heating power was used to validate the CNS performance. The study of the optimal settings for the DPSD data processing to maximize the $n-\gamma$ discrimination capability of the $\mathrm{CNS}$ is reported. The CNS measured both 2.45-MeV and 14-MeV neutrons emitted in AUG D plasmas with a maximum count rate of $5.4 \cdot 10^{5} \mathrm{~s}^{-1}(>10$ times higher than similar spectrometers previously achieved) with an efficiency of $9.3 \cdot 10^{-10}$ events per AUG neutron.
\end{abstract}




\section{Introduction}

Neutron spectrometry is a diagnostic technique that allows the determination of velocity distributions and temperature of plasma ion populations through the analysis of the measured neutron spectrum. The neutrons are produced in nuclear fusion reactions involving hydrogen isotopes ions, deuterons (d) and tritons (t). In general auxiliary heating systems inject power in the plasma altering its thermal conditions. For instance, Neutral Beam Injection (NBI) creates a significant suprathermal ions' tail, increasing the fusion reaction probability as the cross section is higher at larger kinetic energies of the reactants. Thus, the reactants in the plasma will be characterized by different velocity distributions [1][2]. Depending on the velocity of both reactants, the energy spectrum of the outcoming neutron experiences a Doppler broadening in the center of mass frame and an additional Doppler shift in the lab frame [3]. The determination of the kinetic energy of the different reactant ion populations of the plasma is important to prove the effects and the quality of the auxiliary heating schemes applied [2][4]. Moreover, it provides an estimate for the confinement time of the suprathermal ions. A high amount of fast ions can destabilize/stabilize MHD modes and increase the efficiency of Neutral Beam Current Drive. A neutron counter, alone, measures only the coupled effects of the amount of fast ions and their energy. Finally, synergy effects have been observed on several tokamaks between NBI and Ion Cyclotron Heating in the Radio Frequency range, resulting in an anomalously high neutron rate. A measurement of the velocity distribution would allow validating the theoretical models for this synergy [5].

With this aim, a compact neutron spectrometer (CNS) was installed at ASDEX Upgrade (AUG) at IPP Garching (Germany) in November 2008. It is the first neutron 
spectrometer operational there. The instrument consists of a detector designed at the Physikalisch-Technische Bundesanstalt (PTB) Braunschweig (Germany) [6] based on Bicron BC501A liquid scintillating material and a new digital pulse shape discrimination acquisition (DPSD) system designed by ENEA Frascati [7]. The scintillating liquid is contained in a cylindrical cell and is sensitive both to neutrons of energies $>1.5 \mathrm{MeV}$ and to $\gamma$ radiation which give rise to signals of different pulse shapes [8]. This detector and the DPSD system were tested and assembled at PTB.

A similar type of neutron detector was operational with an analog acquisition system at the Joint European Torus (JET) (Culham, England) during the Trace-Tritium Experiment (TTE) in 2003 [4][9]. The spectrometer implemented a control and monitoring system (C\&M) based on a light emitting diode (LED) to cope with the gain variations of the photomultiplier tube (PMT) induced by count rate variations. The correction of the data was performed off-line. Though the spectrometer showed good reliability and performed well during the TTE campaign where it measured 14-MeV neutrons from $d(t, n) \alpha$ fusion reactions, its count rate was limited to a maximum of about $3 \cdot 10^{4} \mathrm{~s}^{-1}$ due to the analog pulse shape discrimination system.

To overcome the count rate limit of the neutron spectrometer, the DPSD system has replaced the analog system. The DPSD system is capable of acquisition up to count rates of the order of $10^{6} \mathrm{~s}^{-1}$, which enables to make use of the fast signal characteristics of BC501A detector. The events are digitized and recorded as pulse shapes, which are analyzed off-line with the DPSD built-in data processing mode using the two-gate method to discriminate neutron and $\gamma$ radiation [10]. The DPSD system is a big step 
forward with respect to the analog acquisition system which recorded the data already as pulse height spectra (PHS).

The new high count rate capability together with the compactness of this type of neutron spectrometer make it of great interest in view of ITER, the new fusion experiment under construction at Cadarache (France).

The paper reports on the CNS assembled and tested at PTB and on the first results attained after the installation at AUG. The detector components, the C\&M and the DPSD systems are described in Section 2. Section 3 reports on the detector tests and calibration to define the HV settings and on its installation and set-up at AUG.

The final settings of the DPSD acquisition and processing modes were defined through the analysis of the data the spectrometer recorded in high power plasma discharges produced at AUG (Section 4). With the optimal parameters for the data processing, the measured pulse shapes were analyzed and the results are reported in Section 5 .

The paper presents the performance of the CNS at AUG in terms of PHS of neutron and $\gamma$ radiations only but not in terms of neutron energy spectra. The CNS provides an integral measurement of the detected events, which results in a continuous PHS up to the maximum energy deposited in the scintillating liquid. Unfolding techniques and an accurate knowledge of the response function are necessary to determine the correspondent neutron energy spectra [11]. The full characterization of the CNS, i.e., the determination of its response function, will be performed at a later stage of the project. 


\section{The CNS system}

The CNS system consists of the detector, the C\&M and the DPSD systems, which are described in the following paragraphs.

\subsection{The BC501A detector}

The CNS makes use of Bicron BC501A liquid scintillating material [12]. The light emission features a maximum wavelength of $425 \mathrm{~nm}$ and short, medium and long components of $3.16 \mathrm{~ns}, 32.3 \mathrm{~ns}$ and $270 \mathrm{~ns}$ decay time, respectively [12]. It is sensitive to neutron and $\gamma$ radiations which excite the organic BC501A molecules at different levels producing pulse shapes with different trailing edges [8]. The detector cell is a rectangular cylinder of $50.8 \mathrm{~mm}$ diameter and height. It is coupled to a PMT through a light guide, shaped as a truncated cone of $52 \mathrm{~mm}$ diameter towards the detector cell and $47.4 \mathrm{~mm}$ diameter towards the photocathode of the PMT. The light guide is partially coated with white paint to assure independence of the light collection efficiency from the interaction point of the radiation in the detector cell [13]. The PMT, Photonis XP 2020 SN, features a passive divider, two different stages with parallel resistor chains, 12 dynodes in total, with capacitors for the last 4 stages and was selected as the best out of ten with respect to the homogeneity of the photocathode response [14][15]. The PMT maximum HV is $2600 \mathrm{~V}$. The photocathode, $51 \mathrm{~mm}$ diameter, is bialkali A type with a maximum light sensitivity at about $400 \mathrm{~nm}$. The PMT is coated with a cylindrical $\mu$-metal cage, $140 \mathrm{~mm}$ long, the diameter being $60 \mathrm{~mm}$ and the thickness $1 \mathrm{~mm}$, to reduce the effect of external magnetic fields on the electron trajectories in the multiplication stage. 


\subsection{The control and monitoring system}

The light yield of BC501A liquid scintillating material is sensitive to temperature variations and the gain of the PMT is affected by high count rate variations. To cope with these effects a C\&M system has been implemented in the CNS system. It consists of a LED based on GaAsP on GaP emitting yellow light (580 nm wavelength). The LED light emission is also sensitive to temperature changes. The C\&M system is adjusted internally in such a way that all temperature effects cancel out for the assembled measurement system [15]. The LED light is beamed directly into the detector cell for mimicking the path and conditions of the light emission induced by radiation interaction and to illuminate the entire photocathode surface. Two potentiometers power the LED and define its intensity and pulse shape [15]. The pulsing frequency is set at $1 \mathrm{kHz}$. This can be modified according to the requirements. The LED signals feature a pulse shape that is well recognized and discriminated from the neutron and $\gamma$ radiation events and used to correct the pulse height of the radiation signals within programmable time intervals $\Delta t$ of the data set in DPSD processing mode off-line.

The light guide, the PMT and the LED of the C\&M system are placed in a cylindrical casing made of aluminum which makes the instrument compact, portable and easy to handle.

A ${ }^{60} \mathrm{Co}$ source is periodically used to check the stability of the spectrometer settings and its absolute energy calibration. It is planned to install permanently a ${ }^{207} \mathrm{Bi}$ source in front of the BC501A cell for the same purpose. 


\subsection{The DPSD system}

ENEA Frascati designed the DPSD system [7][16]. It is based on two $100 \mathrm{MHz}$ AnalogTo-Digital Converters (ADC), a Field Programmable Gate Array (FPGA) and a high speed PCI board (maximum throughput $80 \mathrm{MB} \mathrm{s}^{-1}$ ). It can be operated in acquisition and processing mode. In acquisition mode one can specify parameters such as the triggering threshold, the number of pre-trigger samples and the pulse length. Each triggering event is sampled at $200 \mathrm{MHz}$ by the two ADCs working in interleaved mode (5 ns shift). The pulse shapes are corrected for individual ADCs offset in real time. The dynamic range of the ADCs is 14 bits.

A dynamic window data acquisition module is programmed in the FPGA to terminate the sampling of the pulse shape when its trailing edge reaches the baseline regardless of the pulse length selected as input [7]. The pulse is then stored avoiding the collections of a number of zeros in the pulse tail as it happens in case of a fixed acquisition pulse window. This solution saves memory and raises the number of events recordable (i.e., high count rate capability). Once the measurement is completed, the data set consists in the digitized pulse shapes with information on their trigger time and pulse length.

The off-line DPSD processing mode is used to analyze the data to discriminate neutron and $\gamma$ events and to produce neutron and $\gamma$ PHS and count rates. Each pulse shape is offset-reduced according to a certain number of samples $\mathrm{N}_{\mathrm{o}}$ chosen in the pre-trigger region specified as input of the processing mode. This is to avoid the influence of baseline fluctuations. The pulse shapes of the data set are processed as a sequence in time intervals of selectable duration $\Delta t$ during which the LED pulses are recognized and used to correct the radiation events in the same interval. The analysis software implements the 
short (S) and long (L) gate method [10] which consists in calculating the $Q_{\mathrm{S}} / Q_{\mathrm{L}}$ distribution of the areas $Q_{\mathrm{S}}$ and $Q_{\mathrm{L}}$ of the pulse within the selected $\mathrm{S}$ and $\mathrm{L}$ gates to discriminate neutron and $\gamma$ events (see pulse 583818 in Fig. 5). Once the events are discriminated, they are used to produce neutron and $\gamma$ PHS, i.e., the distribution of the total area of the pulse $Q_{\mathrm{T}}$ within the total (T) gate which is equal to the pulse length. The count rates of neutron and $\gamma$ radiation, of LED and pile up events are also provided. The DPSD system is programmed in LabView ${ }^{\mathrm{TM}}$ and run from a personal computer (PC).

The use of a filter for removing the high frequency components of the PMT anode signal and amplifying the outcome was found beneficial for improving the $n-\gamma$ discrimination [16]. The filter was designed at PTB and it is of $7^{\text {th }}$ order with $-3 \mathrm{~dB}$ at 30 MHz. It reduces the effect of aliasing due to the DPSD sampling rate and amplifies the signal in order to operate the PMT at a lower HV, i.e., in linear regime, to minimize the effects induced by high count rate variations [16]. An amplification of 4 was found to be a good trade off between the PMT HV and the exploitation of the DPSD system input voltage range of $\pm 2.8 \mathrm{~V}$.

\section{CNS settings and installation at AUG}

The CNS was tested using a ${ }^{207} \mathrm{Bi}$ source, which emits $\gamma$-rays of $0.569 \mathrm{MeV}, 1.064 \mathrm{MeV}$ and $1.771 \mathrm{MeV}$, corresponding to Compton edges at $0.395 \mathrm{MeV}, 0.865 \mathrm{MeV}$ and 1.555 $\mathrm{MeV}$ in the CNS detector, respectively [11]. Their positions provide useful information for setting the working points of the spectrometer (i.e., the HV of the PMT) and for defining the energy calibration. The quality of the detector performance is established by comparing the Compton edges to the corresponding ones measured with well- 
characterized spectrometers of the same type in use at PTB as well as with Monte Carlo calculations. The full characterization of the CNS will be obtained at a later stage by exposing the instrument to well-defined neutron beams in terms of energy and fluence at the PTB accelerator. This will make the measurement of the CNS response function possible which will then be use to unfold the neutron PHS to extract the correspondent neutron energy spectrum [11].

Fig. 1 shows the results of the fitting routine used for the analysis of the Compton edges of the LED C\&M corrected PHS obtained exposing the CNS to the ${ }^{207} \mathrm{Bi}$ source. From the fit, the resolution in terms of full width at half maximum (FWHM) is calculated [16]. Typical values for the resolution of the ${ }^{207} \mathrm{Bi}$ Compton edges are $25-30 \%$ for the first, $16-20 \%$ for the second and $13-16 \%$ for the third one for a well designed detector. The values for the detector described in [11] using this method are $28 \%$ for the first Compton edge, $20 \%$ for the second and $14 \%$ for the third one. In case of the CNS for AUG it resulted $27 \%, 17 \%$ and $13 \%$, respectively. From the Compton energy of $\gamma$ radiation deposited in the $\mathrm{BC} 501 \mathrm{~A}$ detector cell, information upon that due to neutrons can be estimated through the comparison of the light yield. This allows defining the HV settings of the PMT in order to detect neutrons and the energy scale that corresponds to the measured neutron or $\gamma$ PHS.

At AUG, Deuterium (D) plasmas are studied. Here $d(d, n)^{3} \mathrm{He}, \mathrm{d}(\mathrm{d}, \mathrm{p}) \mathrm{t}$ and $\mathrm{d}(\mathrm{d}, \gamma) \alpha$ nuclear fusion reactions give rise to different products with branching ratios of about 50 $\%$ for the first two and $10^{-3} \%$ for the third one [17][18].

The CNS goal is the study and interpretation of the ion velocity distribution of the reactants that give rise to the measured $2.45-\mathrm{MeV}$ neutron PHS. Since tritons are also 
produced in $\mathrm{D}$ plasmas discharges at $1 \mathrm{MeV}$ energy, they can react with deuterons in $\mathrm{t}(\mathrm{d}, \mathrm{n}) \alpha$ (about $100 \%$ branching ratio) and produce neutrons of energy $\geq 14.03 \mathrm{MeV}$. This process is called triton burn up [19]. The CNS is also sensitive to these neutrons, which signals can be discriminated from the ones of $2.45-\mathrm{MeV}$ neutrons and from $\gamma$ radiation. At AUG, the CNS dymanic range (i.e., energy bite) was set to measure dt neutron PHS as well. For a dedicated $2.45-\mathrm{MeV}$ neutron measurement, another setting can be adopted (i.e., the PMT gain becomes a factor of 2 higher by changing the HV from $-1580 \mathrm{~V}$ to $1680 \mathrm{~V})$.

The DPSD system can be used for count rates up to $10^{6} \mathrm{~s}^{-1}$, which induce PMT gain variations of about $30 \%$ [16]. Lower PMT HV is beneficial to minimize this effect. Tests were carried out to determine the linearity region of PMT as a function of the HV [15]. The final HV settings of the PMT adopted for the CNS at AUG is $-1550 \mathrm{~V}$ (i.e., much lower than the maximum HV of $-2600 \mathrm{~V})$. Stability tests of the CNS were carried out with a ${ }^{207} \mathrm{Bi}$ source and provided results in terms of resolution as specified before.

The CNS system is shown in Fig. 2(a) as it is installed at AUG. It is about 50-cm long and the detector cell is visible on the left in front of the neutron collimator obtained in the concrete wall. The signal cable is super-screened to reduce the noise contamination from the tokamak environment. The HV module, the LED C\&M unit, the PTB custom-made filter and the DPSD system are placed in a cubicle in the AUG control room, about $50 \mathrm{~m}$ away from the spectrometer. The anode signal of the PMT is plugged into the PTB filter which output is fed into the DPSD system to be read out.

The CNS is viewing the plasma radially in the equatorial plane of the tokamak at about $11 \mathrm{~m}$ distance from the plasma center (Fig. 2(b)) through a pre-collimator and a 
collimator. The pre-collimator is a polyethylene cube of $60 \mathrm{~cm}$ side length. It has an opening of $8.8 \mathrm{~cm}$ in the center and it is placed at a distance of about $4.8 \mathrm{~m}$ from the plasma centre. The collimator is a cylindrical opening in the $2 \mathrm{~m}$ thick concrete wall shielding the tokamak. The diameter is $7.6 \mathrm{~cm}$, a bit wider than the diameter of the detector cell. The CNS integral line of sight radially covers about $132 \mathrm{~cm}^{2}$ of the plasma core at the toroidal magnetic axis.

The first tests of the CNS exposed to the neutron emission of the plasma resulted in a $\mathrm{n} / \gamma$ count rate ratio equal to 1 . To verify whether the $\gamma$ radiation was coming directly from the plasma through the collimators or it was induced by neutron interactions in the tokamak structure, i.e., $\gamma$ background, an extra layer of lead bricks was built to cover the pre-collimator cube face around its opening towards the spectrometer. This resulted in $n / \gamma$ ratio equal to 10 , which demonstrated the effectiveness of the shielding in reducing the contribution of the $\gamma$ background (see later).

The CNS has been tested in plasma experiments to verify its performance and the quality of the measurements. The CNS data acquisition was started manually for $120 \mathrm{~s}$ when the AUG discharge was announced. Fig. 3 shows the results in terms of count rates and average LED pulse area $\left\langle Q_{T}^{L E D}>\right.$ measured during AUG plasma discharge 23056. The pulse area of the LED C\&M signals recorded within the time interval $\Delta t$ (i.e., 100 LED pulses for $\Delta t=100 \mathrm{~ms}$ ) is averaged to improve its accuracy and used for the correction of the radiation pulse shapes. The total, neutron and $\gamma$ count rates are displayed as a function of DPSD acquisition time. The difference between the total count rate and the sum of neutron and $\gamma$ count rates is due to pile up events, which are recognized and 
rejected in the DPSD processing mode. Their contribution is of the order of $6 \%$ for $1.2 \cdot 10^{5} \mathrm{~s}^{-1}$ count rate.

The average LED pulse area $<Q_{T}^{L E D}>$ follows the trend of the count rate only roughly and with opposite sign with a variation of $15 \%$, although from experience high count rates increase the gain of the PMT [15][16]. The trend of $\left\langle Q_{T}^{L E D}>\right.$ in Fig. 3 is the result of magnetic field variations during the plasma discharge, which the spectrometer is exposed to in front of the collimator. These variations have an impact on the PMT behavior. The magnetic field was measured in different orientations at the detector cell position during few plasma discharges and variations of the order of $1.8 \mathrm{mT}$ were observed. To cope with such effects, the detector was inserted in a $\mu$-metal cylinder of $110 \mathrm{~mm}$ diameter, $350 \mathrm{~mm}$ long and $1 \mathrm{~mm}$ thick. Meca Magnetic manufactured the $\mu-$ metal shielding with a suppression factor of $10^{4}$ [20]. The beneficial effects of the shielding are displayed in Fig. 4 where now $\left\langle Q_{T}^{L E D}>\right.$ measured during AUG plasma discharge 23069 provides the information on true behavior of the PMT induced only by the count rate (3\% maximum variation) and allows for an effective LED correction of the data.

With this modification the CNS was operational and used to measure the neutron emission from D plasma experiments at AUG.

\section{Analysis of the DPSD performance}

The performance of the DPSD system was investigated using AUG discharge 24151 featuring 7.3 MW of NBI and 1.5 MW of Electron Cyclotron Resonant Heating (ECRH). The parameters of the plasma discharge are presented in Table 1. The neutron emission 
data of the discharge starts at $68.6 \mathrm{~s}$ of the DPSD acquisition time. The discharge lasted $6.4 \mathrm{~s}$. The threshold of the DPSD acquisition mode was $10 \mathrm{mV}$ with $\mathrm{N}=6$ pre-trigger samples for the pulse shapes of the triggering events. The study of the DPSD performance is performed with the method presented in [21] and described in details in [22]. Here we report only on the results.

Table 1: Main parameters of discharge 24151 at AUG.

\begin{tabular}{|l|c|}
\hline Plasma current / MA & 0.8 \\
\hline Electron density / m & -3 \\
\hline Toroidal magnetic field / T & $6.0 \cdot 10^{19}$ \\
\hline Safety factor q95 & -2.4 \\
\hline Stored energy / MJ & 4.7 \\
\hline Central electron temperature / keV & 0.4 \\
\hline ECRH / MW & 3.0 \\
\hline Total NBI power / MW & 1.5 \\
\hline
\end{tabular}

\subsection{Validation of the DPSD acquisition mode}

The full data set of digitized pulse shapes of the events recorded during the discharge was analyzed. Examples of pulse shapes with their corresponding number as recorded in the data set are shown in Fig. 5 with the indication of the threshold level $(10 \mathrm{mV}$ correspond to $29 \mathrm{ADC}$ channels, i.e., $0.34 \mathrm{mV} / \mathrm{ADC}$ channels) adopted in the measurement, the pretrigger region and the $\mathrm{S}, \mathrm{L}$ and $\mathrm{T}$ gates used later on in the DPSD processing mode to integrate the pulse shapes for the $n-\gamma$ discrimination. The time difference between pulse samples is $5 \mathrm{~ns}$.

The total number of events acquired during $120 \mathrm{~s}$ is 2397349 of which $92 \%$ correspond to single event pulse shapes (see Fig. 5) used then for the optimization of the DPSD processing mode settings. The other $8 \%$ of the events recorded were identified as 
pile-ups (5\%) and corrupted pulse shapes (see [22] for details). Pile up events, mostly recorded during the discharge (see Section 5), are recognized in the analysis method and can also be further re-processed to extract information on the nature of the incoming radiation $[21][23]$.

\subsection{Optimal settings for the $n-\gamma$ discrimination with the DPSD processing mode}

The parameters for the DPSD processing mode were chosen to maximize the $n-\gamma$ discrimination capability of the CNS with the two-gate method [10]. This study concerns the offset reduction and LED C\&M correction of the data, and the choice of the optimal gates $\mathrm{S}$ and L [21][22].

The offset reduction of the data consists in calculating the average amplitude of the pulse shape for a certain number of samples $\mathrm{N}_{\mathrm{o}}$ in the pre-trigger region $\mathrm{N}\left(\mathrm{N}_{\mathrm{o}} \leq \mathrm{N}\right)$. This value is then subtracted from the amplitude value of each sample of the pulse shape. Since the calculated offset value is subject to an uncertainty, after the reduction this uncertainty will propagate to the $Q_{\mathrm{S}}, Q_{\mathrm{L}}$ and $Q_{\mathrm{T}}$ values proportionally to the number of samples used for the $\mathrm{S}, \mathrm{L}$ and $\mathrm{T}$ gates and will affect the quality of the $\mathrm{n}-\gamma$ discrimination. A Bayesian method was applied to determine the optimal number of samples $\mathrm{N}_{0} *$ which gives rise to the offset value with the lowest uncertainty. The method allows for the determination of the offset uncertainty distribution $U_{o}$ as a function of the number of samples $\mathrm{N}_{\mathrm{o}}$ for the offset calculation: $\mathrm{U}_{\mathrm{o}}=\left(\sqrt{\frac{\sum_{\mathrm{i}=1}^{\mathrm{N}_{\mathrm{o}}}\left(v_{\mathrm{i}}-b\right)^{2}}{\mathrm{~N}_{\mathrm{o}}\left(\mathrm{N}_{\mathrm{o}}-1\right)}}\right) \cdot t_{\mathrm{N}_{\mathrm{o}}-1}$, where $v_{\mathrm{i}}$ is the pulse amplitude of sample $\mathrm{i}, b$ is the offset calculated for $\mathrm{N}_{\mathrm{o}}$ pre-trigger samples and $t_{\mathrm{N}_{\mathrm{o}}-1}$ is the Student's t-distribution on $v=\mathrm{N}_{0}-1$ degrees of freedom (see [21][22] and references 
therein). For each event of the data set, the uncertainty $U_{o}$ was calculated individually. From these data, a set of probability distributions of $U_{o}$ for different values of $N_{o}$ is calculated (Fig. 6). From this it is found that $\mathrm{N}_{\mathrm{o}} *=5$ samples is the optimal choice since it corresponds to the uncertainty distribution with the lowest average uncertainty value of about 5 a.u..

The LED C\&M system monitors the PMT gain during the measurements and provides the important correction factor to the pulse shapes for the determination of the PHS. Particularly, the neutron PHS is the one of interest since it contains the spectroscopic information, which is extracted using the CNS response function and data unfolding techniques [11]. The C\&M data correction makes use of the average LED pulse area $\left\langle Q_{T}^{L E D}>\right.$ calculated in a time interval $\Delta t$ which is compared to a reference LED pulse area $Q_{\mathrm{T}}{ }^{\mathrm{LED}}=3000$ a.u. (see Figs. 1 and 9). Their ratio is used to correct all neutron and $\gamma$ radiation pulse amplitudes within the same time interval $\Delta t[21][22]$. The average LED pulse area $\left\langle Q_{T}^{L E D}>\right.$ featured a $5.2 \%$ variation during the discharge, from 3040 a.u. up to 3196 a.u.. After the discharge, $<Q_{T}^{L E D}>$ decreased to 3027 a.u. due to the afterglow effect of the PMT [16]. The analysis of AUG discharge 24151 data showed that the PHS of neutron and $\gamma$ radiations are the same for LED correction applied within $\Delta t=$ $[50,200] \mathrm{ms}$. These results confirm the reliability of the settings chosen for the PMT HV and of the LED C\&M correction method.

With $\mathrm{N}_{\mathrm{o}} *=5$ samples for the offset reduction and $\Delta t=100 \mathrm{~ms}$ for the LED correction of the data, the analysis of AUG discharge 24151 was carried out to determine the optimal S-L gate combination that maximizes the $n-\gamma$ discrimination [21][22]. Different combinations of $\mathrm{S}$ and $\mathrm{L}$ gates were investigated within the sample number range $[2,120]$ 
with $\mathrm{S}<\mathrm{L}$ to evaluate the $n-\gamma$ separation based on the Figure-Of-Merit (FOM) of the distribution $Q_{\mathrm{S}} / Q_{\mathrm{L}}$ defined as: $\mathrm{FOM}=\frac{\Delta P}{\Gamma_{\mathrm{n}}+\Gamma_{\gamma}}$, where $\Delta P$ is the separation between the neutron and $\gamma$ centroids of the $Q_{\mathrm{S}} / Q_{\mathrm{L}}$ distribution of FWHMs $\Gamma_{\mathrm{n}}$ and $\Gamma_{\gamma}$ [24][25]. The optimal choice of S-L gates corresponds to the $Q_{\mathrm{S}} / Q_{\mathrm{L}}$ distribution with maximum FOM value as shown in Fig. 7.

The optimal S-L combination of 4-15 samples was found to maximize the FOM value. These two gates correspond to time durations of $20 \mathrm{~ns}$ and $75 \mathrm{~ns}$, respectively, which means that to optimize the $n-\gamma$ discrimination capability of the BC501A detector DPSD system only the short and medium decay time components of its light emission are the ones to be exploited. This possibility improves even more the count rate capabilities of the CNS since the dynamic window data acquisition module of the DPSD system can be set to record a lower number of samples for each triggering pulse shape [16][21][22].

With the optimal gates, all the pulse shapes of the data set were re-processed to produce the contour plot of $Q_{\mathrm{S}} / Q_{\mathrm{L}}$ vs. $Q_{\mathrm{T}}$ presented in Fig. 8. This shows different regions, which correspond to the different incoming radiations measured with the CNS. The distribution of the $2.45-\mathrm{MeV}$ neutrons generated in AUG plasma discharge 24151 is visible for $Q_{\mathrm{S}} / Q_{\mathrm{L}}=[0.50,0.79]$. The $\gamma$ radiation instead falls within $Q_{\mathrm{S}} / Q_{\mathrm{L}}=[0.82,1.00]$ while a trace of the 14-MeV neutrons as a results of the triton burn up process described in Section 3 is visible for $Q_{\mathrm{S}} / Q_{\mathrm{L}}=[0.70,0.82]$.

As a result of this analysis, specific type of radiation from different regions of interest of Fig. 8 can be selected and compared. Table 2 summarizes the total number of events corresponding to different regions of Fig. 8 from where the pulse shapes shown in Fig. 5 
were selected. These pulse shapes are now identified in Table 2 in terms of their $Q_{\mathrm{S}} / Q_{\mathrm{L}}$ and $Q_{\mathrm{T}}$ values.

Table 2: Total number of events within ranges of $Q_{\mathrm{S}} / Q_{\mathrm{L}}$ and $Q_{\mathrm{T}}$ from Fig. 8 corresponding to neutron and $\gamma$ events of different energies. The examples of pulses shown in Fig. 5 fall into different parts of the $Q_{\mathrm{S}} / Q_{\mathrm{L}}$ vs. $Q_{\mathrm{T}}$ plot and are stated in the table with their $Q_{\mathrm{S}} / Q_{\mathrm{L}}$ and $Q_{\mathrm{T}}$ values.

\begin{tabular}{|c|c|c|c|c|c|c|}
\hline $\begin{array}{c}\text { Range of } \\
Q_{\mathrm{S}} / Q_{\mathrm{L}}\end{array}$ & $\begin{array}{c}\text { Range of } \\
Q_{\mathrm{T}} / \text { a.u. }\end{array}$ & $\begin{array}{c}\text { Total } \\
\text { events }\end{array}$ & $\begin{array}{c}\text { Radiation } \\
\text { type }\end{array}$ & $\begin{array}{c}\text { Pulse } \\
\text { number }\end{array}$ & $Q_{\mathrm{S}} / Q_{\mathrm{L}}$ & $Q_{\mathrm{T}} /$ a.u. \\
\hline$[0.50,0.79]$ & {$[0,300]$} & 1874501 & $2.45-\mathrm{MeV}$ 's & No. 73173 & 0.70 & 168.32 \\
\hline$[0.82,1.00]$ & {$[0,1800]$} & 162931 & $\gamma$ 's & No. 193140 & 0.84 & 1419.19 \\
\hline$[0.79,0.82]$ & {$[0,300]$} & 33802 & n's or $\gamma$ 's & No. 8337 & 0.79 & 51.46 \\
\hline$[0.70,0.82]$ & {$[300,1800]$} & 2256 & $14-\mathrm{MeV}$ n's & No. 583818 & 0.79 & 1467.97 \\
\hline
\end{tabular}

The $2.45-\mathrm{MeV}$ and $14-\mathrm{MeV}$ neutron pulse shapes 73173 and 583818, respectively, correspond to neutron interactions in BC501A detector cell, which deposit their full energy. Furthermore, the analysis can be focused on ranges of $Q_{\mathrm{T}}$ of interests for which the optimal S-L combination can be newly calculated for improving the $n-\gamma$ separation in that specific range. This might help to better discriminate the events in the $n-\gamma$ overlapping region [21][22].

The analysis of the DPSD performance provided a deeper insight in the data set to validate the DPSD acquisition mode and to determine the optimal input parameters for the processing mode. These parameters can now be used for the systematic analysis of all the data recorded with the CNS at AUG. 


\section{Results on the performance of the CNS at AUG}

The analysis of the full data set of AUG discharge 24151 was carried out with the DPSD processing mode to produce PHS and count rates. This to quantify the performance of the CNS system in terms of neutron and $\gamma$ sensitivity with respect to the AUG total neutron yield and of count rates achieved. When the CNS response function will be available the unfolding of the neutron PHS will produce the neutron energy spectrum which shape reflects the velocity distributions of the reactants in D plasma [1][2][4][11].

The PHS of the total events together with the ones due to neutron and $\gamma$ and LED events are shown in Fig. 9. The LED $Q_{\mathrm{T}}$ distribution is peaked about the reference area $Q_{\mathrm{T}}{ }^{\mathrm{LED}}{ }^{*}=3000$ a.u. used for the C\&M data correction. The neutron PHS is the result of the contributions of $2.45-\mathrm{MeV}$ neutrons from dd reactions, which maximum energy deposition corresponds to $Q_{\mathrm{T}} \approx 200$ a.u., and of $14-\mathrm{MeV}$ neutrons from dt reactions up to $Q_{\mathrm{T}} \approx 1400$ a.u.. The triton burn up contribution is about $0.3 \%$ of the total measured neutron emission. The CNS capability of simultaneously measuring the full neutron energy emission from D plasmas is thus demonstrated. For high auxiliary heated plasmas, a detailed analysis of the neutron energy spectrum and of the resulting ion velocity distributions may provide information on the densities of the $\mathrm{d}$ and $\mathrm{t}$ ion populations [1]. According to the energy calibration of Fig. 1, the $\gamma$ PHS shows some Compton edges at energies of about $0.36 \mathrm{MeV}, 0.53 \mathrm{MeV}$ and $0.75 \mathrm{MeV}$ and one up to $Q_{\mathrm{T}} \approx 1235$ a.u. which corresponds to the energy deposition of $2.2-\mathrm{MeV} \gamma$ 's produced in $\mathrm{p}(\mathrm{n}, \mathrm{d}) \gamma$ reactions neutrons induce in the polyethylene pre-collimator.

The pile up and single event count rates are determined together with the ones corresponding to total, neutron, $\gamma$ and LED signals. The total, neutron and $\gamma$ count rates 
are shown in Fig. 10 and compared to the $100 \mathrm{~ms}$ time averaged AUG total neutron yield $<\mathrm{Y}_{\mathrm{n}}{ }^{\text {TOT }}>$ measured with $1 \mathrm{~ms}$ time resolution by the ${ }^{238} \mathrm{U}$ fission chambers of the AUG neutron monitor system, which also consists of $\mathrm{BF}_{3}$ and ${ }^{3} \mathrm{He}$ proportional counters and ${ }^{235} \mathrm{U}$ fission chambers [26]. The results of the analysis are presented in Table 3.

Table 3: Summary of the results the CNS obtained during AUG discharge 24151 in terms of events and count rates for each type of event (see Fig. 10).

\begin{tabular}{|l|c|}
\hline AUG $\mathrm{Y}_{\mathrm{n}}^{\text {TOT }}$ & $2.5 \cdot 10^{15}$ \\
\hline AUG $\mathrm{Y}_{\mathrm{n}}^{\mathrm{MAX}} / \mathrm{s}^{-1}$ & $9.1 \cdot 10^{14}$ \\
\hline Neutron events & $1.9 \cdot 10^{6}$ \\
\hline Maximum count rate $/ \mathrm{s}^{-1}$ & $5.4 \cdot 10^{5}$ \\
\hline Maximum neutron count rate $/ \mathrm{s}^{-1}$ & $4.2 \cdot 10^{5}$ \\
\hline Average pile up count rate $/ \mathrm{s}^{-1}$ & $4.7 \cdot 10^{4}$ \\
\hline Average single count rate $/ \mathrm{s}^{-1}$ & $3.7 \cdot 10^{5}$ \\
\hline Average single/pile up ratio & 7.9 \\
\hline Average neutron count rate $/ \mathrm{s}^{-1}$ & $3.4 \cdot 10^{5}$ \\
\hline Average $\gamma$ count rate $/ \mathrm{s}^{-1}$ & $3.3 \cdot 10^{4}$ \\
\hline Average $\mathrm{n} / \gamma$ ratio & 10.3 \\
\hline
\end{tabular}

The total neutron yield $\mathrm{Y}_{\mathrm{n}}{ }^{\text {TOT }}$ measured for AUG discharge 24151 is $2.5 \cdot 10^{15}$ neutrons with a maximum rate of $9.1 \cdot 10^{14} \mathrm{~s}^{-1}(1 \mathrm{~ms}$ average $)$. The CNS total efficiency to neutron radiation during the discharge can be calculated by comparing the number of events in the PHS to $\mathrm{Y}_{\mathrm{n}}^{\text {TOT }}$ and it results $7.6 \cdot 10^{-10}$.

In AUG discharge 24151, the CNS achieved a maximum count rate of $5.4 \cdot 10^{5} \mathrm{~s}^{-1}$ of which $4.2 \cdot 10^{5} \mathrm{~s}^{-1}$ is due to neutrons $(78 \%)$. This represents a break-through with respect to the limit of $3 \cdot 10^{4} \mathrm{~s}^{-1}$ a similar neutron spectrometer attained at JET during the TTE campaign. 
The single/pile up event ratio during the discharge is 7.9. As noted before, the pile up events are rejected in this analysis but, since they are tagged, they can be further analyzed to extract information on the nature of the incoming radiation and, thus, increasing the number of neutron and $\gamma$ events recorded [21][23].

An average $\mathrm{n} / \gamma$ count rate ratio of 10.3 was measured in AUG discharge 24151 compared to 1 obtained before the installation of the lead shielding, which demonstrates the quality of the CNS set-up for $\gamma$ background suppression.

Concerning the average pulse height of the LED events $\left\langle Q_{T}^{L E D}>\right.$ a maximum variation of $6.6 \%$ was measured during the discharge. This is entirely due to the gain variation of the PMT induced by high count rates since the magnetic effects are suppressed by the external $\mu$-metal shielding.

\section{Conclusions}

The paper presented the tests of the new compact neutron spectrometer and the procedures used for the optimal choice of its settings under real measurement conditions after the installation at AUG in November 2008. The CNS was set to measure both 2.45$\mathrm{MeV}$ and 14-MeV neutrons emitted from D plasmas. The CNS was exposed to the neutron emission from a high auxiliary power discharge at AUG where it reached a maximum count rate of $5.4 \cdot 10^{5} \mathrm{~s}^{-1}$ of which $4.2 \cdot 10^{5} \mathrm{~s}^{-1}$ is due to neutrons.

A detailed study was carried out to validate the measured data and to determine the optimal parameters for the two-gate analysis method implemented in the DPSD processing mode. It yields the optimal $n-\gamma$ discrimination capability using gates of $20 \mathrm{~ns}$ 
and 75 ns duration, respectively, thus just making use of the characteristic short and medium decay time components of the light generated in the BC501A scintillating liquid.

The CNS sensitivity to the full neutron emission of D plasmas was demonstrated since both $2.45-\mathrm{MeV}$ dd and $14-\mathrm{MeV}$ dt neutrons were measured. The spectrometer response function, to be measured at $\mathrm{PTB}$, will enable the determination of the neutron energy spectra and its analysis in terms of velocity distributions and temperatures of the plasma ion populations.

The high count rate capability the CNS demonstrated at AUG represents a breakthrough with respect to what previously achieved with organic liquid scintillation neutron spectrometers.

\section{Acknowledgments}

The authors would like to thank M. Weierganz and M. Reginatto for the valuable discussion and expertise, Associazione Euratom-ENEA sulla Fusione (F. Belli, D. Marocco, B. Esposito and M. Riva) for providing the DPSD system and for their collaboration, and ASDEX Upgrade Team for their contribution to the success of the installation, tests and experiments.

\section{References}

[1] L. Giacomelli, S. Conroy, G. Ericsson, G. Gorini, H. Henriksson, A. Hjalmarsson, J. Källne, and M. Tardocchi, Eur. Phys. J. D 33, 235-241 (2005).

[2] L. Bertalot, S. Conroy, A. Murari, M. Reginatto, H. Schuhmacher, A. Zimbal and JET-EFDA Contributors, $32^{\text {nd }}$ EPS Conference on Plasma Phys. Tarragona, 27 June - 1 
July $2005 \quad$ ECA Vol. 29C, P-1.078 http://epsppd.epfl.ch/Tarragona/pdf/P1_078.pdf.

[3] M. Tardocchi, "Neutron emission spectroscopy studies of fusion plasmas of deuterium-tritium in tokamaks", PhD Thesis, Uppsala University (2000).

[4] A. Zimbal, M. Reginatto, H. Schuhmacher, L. Bertalot, B. Esposito, F. Poli, J.M. Adams, S. Popovichev, V. Kiptily, A. Murari and Contributors to the EFDA-JET work program, Rev. Sci. Instrum. 75 (2004), p. 3553.

[5] L. Giacomelli, A. Hjalmarsson, J. Källne, C. Hellesen, M. Tardocchi, G. Gorini, D. Van Eester, E. Lerche, T. Johnson, V. Kiptily, S. Conroy, E. Andersson Sundén, G. Ericsson, M. Gatu Johnson, H. Sjöstrand, M. Weiszflog, Rev. Sci. Instrum. 79, 10E514 (2008).

[6] H. Klein, Radiation Protection Dosimetry 107, Nos 1-3 (2003), pp. 95-109, PMID:

\section{$\underline{14756170 .}$.}

[7] M. Riva, B. Esposito, D. Marocco, $10^{\text {th }}$ ICALEPCS Int. Conf. on Accelerator \& Large Expt. Physics Control Systems Geneva, 10-14 Oct 2005, PO2.041-4 (2005), http://accelconf.web.cern.ch/accelconf/ica05/proceedings/pdf/P3_041.pdf.

[8] G.F. Knoll, "Radiation detection and measurement" (New York: John Wiley\&Sons, Inc.), Third edition 1999, p. 230.

[9] L. Bertalot, J.M. Adams, M. Angelone, S. Conroy, B. Esposito, Y. Kaschuck, H. Henriksson, P. Lamalle, D. Marocco, A. Murari, N. Hawkes, M. Pillon, S. Popovichev, M. Reginatto, M. Riva, H. Schuhmacher, D. Stork, K.-D. Zastrow, A. Zimbal and The JET EFDA Contributors, Fusion Engineering and Design 74, Issues 1-4, November 2005, pp. 835-839. 
[10] S. Marrone, D. Cano-Ott, N. Colonna, C. Domingo, F. Gramegna, E.M. Gonzalez, F. Gunsing, M. Heil, F. K.appeler, P.F. Mastinu, P.M. Milazzo, T. Papaevangelou, P. Pavlopoulos, R. Plag, R. Reifarth, G. Tagliente, J.L. Tain, K. Wisshak, Nucl. Instr. Meth. A 490 (2002), pp. 299-307, doi:10.1016/S0168-9002(02)01063-X.

[11] H. Klein, S. Neumann, Nucl. Instr. Meth. A 476 (2002), p. 132-142, doi:10.1016/S0168-9002(01)01410-3.

[12] See BC501A properties at http://www.detectors.saintgobain.com/uploadedFiles/SGdetectors/Documents/Product_Data_Sheets/BC501-501A519-Data-Sheet.pdf.

[13] H. Klein, H. Schölermann, IEEE Transactions on Nuclear Science 26, Issue 1, Feb. 1979, pp. 373-377.

[14] See Photonis PMT characteristics at http://www.photonis.com/upload/industryscience/pdf/pmt/XP2020.pdf.

[15] K. Tittelmeier, H.J. Barrenscheen, "Stabilization of Scintillation Detectors", $\begin{array}{llll}\text { Laboratory } & \text { Report } & \text { PTB } & \text { 6.42-03-2 }\end{array}$ http://www.ptb.de/en/org/6/65/651/pdf/pmt_laborbericht.pdf.

[16] D. Marocco, F. Belli, B. Esposito, M. Riva, L. Giacomelli, M. Reginatto, K. Tittelmeier and A. Zimbal, IEEE Transactions on Nuclear Science (TNS) 56, Number 3, June 2009, pp. 1168-1173.

[17] H. Henriksson, "Neutron spectroscopy studies of heating effects in fusion plasmas", PhD Thesis, Uppsala University (2003).

[18] F.E. Cecil, H. Liu, and J. S. Yan, Phys. Rev. C 47 (1993), pp.1178-1183, doi: 10.1103/PhysRevC.47.1178. 
[19] G. Gorini, L. Ballabio and J. Källne, Plasma Phys. Control. Fusion 39 (1997), pp. 61-71.

[20] Meca Magnetic Deutschland, http://www.abschirmung.com/accueil_de.php?id=45.

[21] L. Giacomelli, A. Zimbal, M. Reginatto, K. Tittelmeier, Rev. Sci. Instrum. 82, 013505 (2011), doi:10.1063/1.3519016.

[22] L. Giacomelli, A. Zimbal, M. Reginatto, and K. Tittelmeier, PTB Report N-57 Braunschweig, August 2010, ISBN 978-3-86918-121-9 ISSN 0936-0492.

[23] F. Belli, B. Esposito, D. Marocco, M. Riva, Y. Kaschuck, G. Bonheure, JET EFDA contributors, Nucl. Instr. Meth. A 595 (2008), pp. 512-519, doi:10.1016/j.nima.2008.06.045.

[24] G.F. Knoll, "Radiation detection and measurement" (New York: John Wiley\&Sons, Inc.), Third edition 1999, p. 680.

[25] C. Guerrero, D. Cano-Ott, M. Fernández-Ordóñez, E. González-Romero, T. Martínez, D. Villamarín, Nucl. Instr. Meth. A 597 (2008), Issues 2-3, pp. 212-218, doi:10.1016/j.nima.2008.09.017.

[26] C. Höhbauer, "Modellierung der Neutronenproduktion in Plasmaentladungen am Fusionsexperiment ASDEX Upgrade”, IPP 1/339, December 2010, p. 27, http://edoc.mpg.de/520581. 


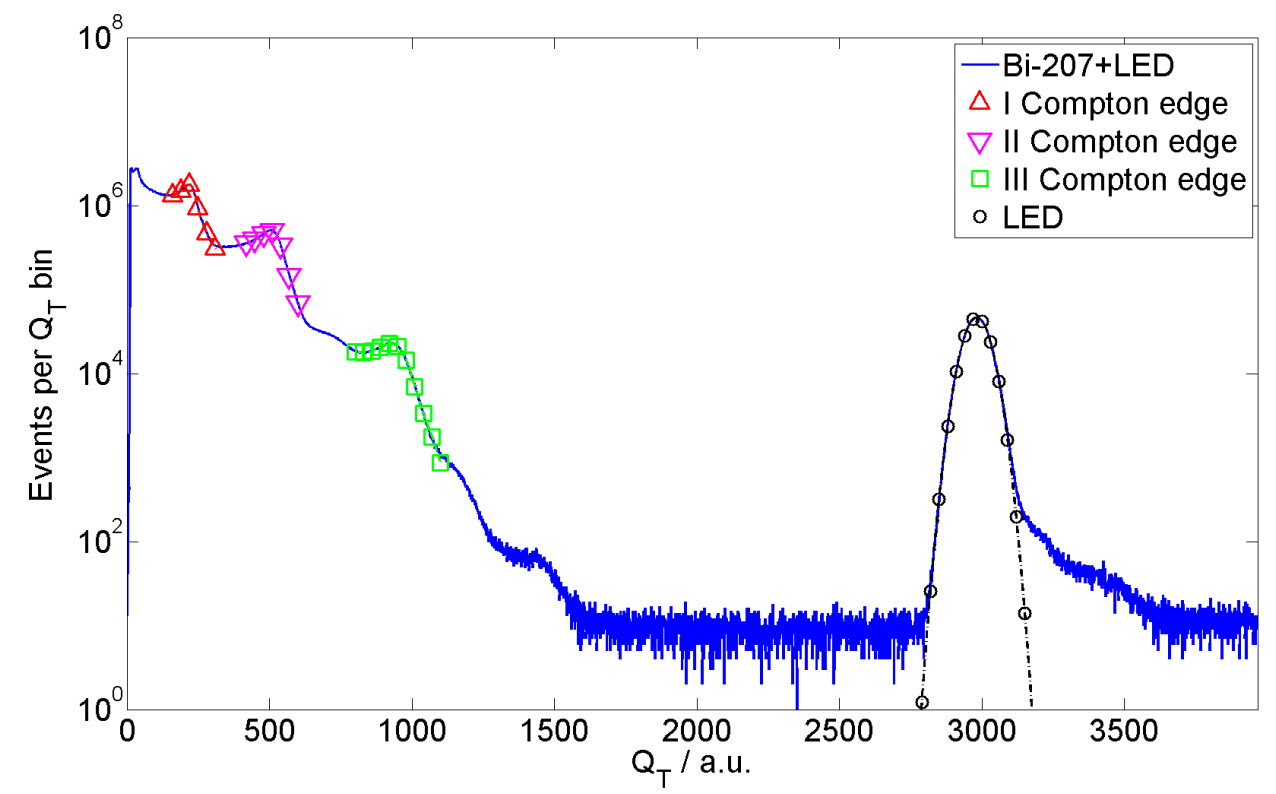

Fig. 1: (Color online) The ${ }^{207} \mathrm{Bi}$ PHS (the distribution of the events as a function of their pulse height $\mathrm{Q}_{\mathrm{T}}$ ) measured with CNS. The Compton edges of the ${ }^{207} \mathrm{Bi} \gamma$-rays and the LED peak have been fitted for assessing the performance of the BC501A liquid scintillating detector of the CNS.
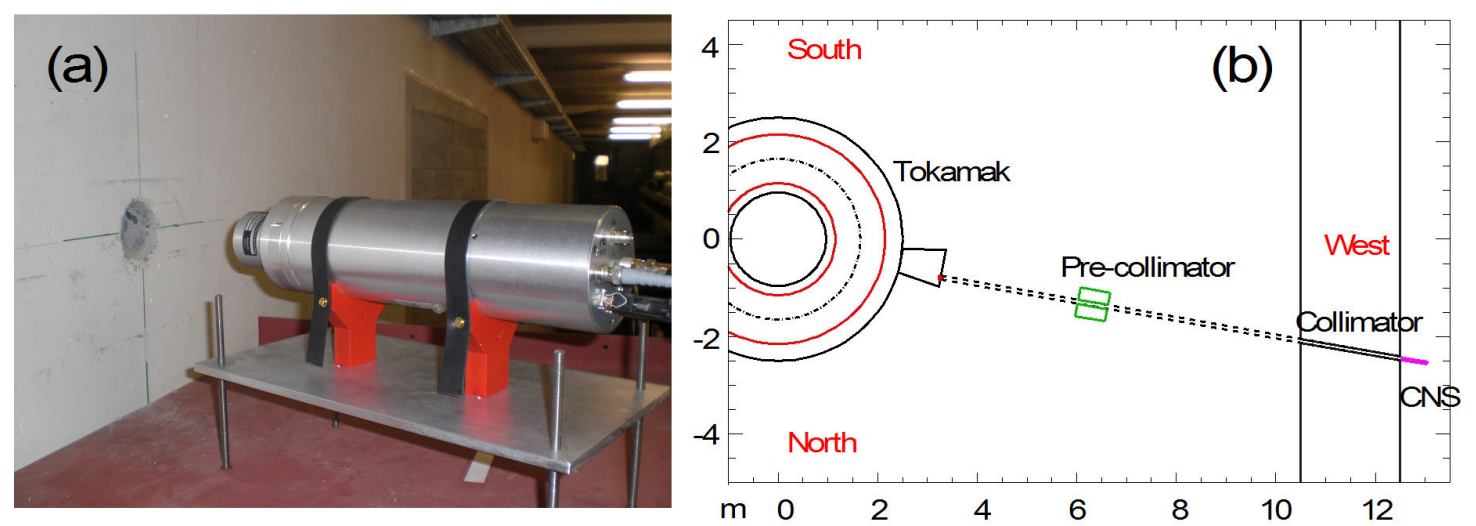

Fig. 2: (Color online) In (a), the CNS installed at AUG in front of the 2-m long neutron collimator obtained in the concrete wall. In (b), schematics of the CNS line of sight from above. The CNS is viewing the plasma radially in the tokamak equatorial plane at a distance of about $11 \mathrm{~m}$ from the plasma center through a pre-collimator and a collimator. 


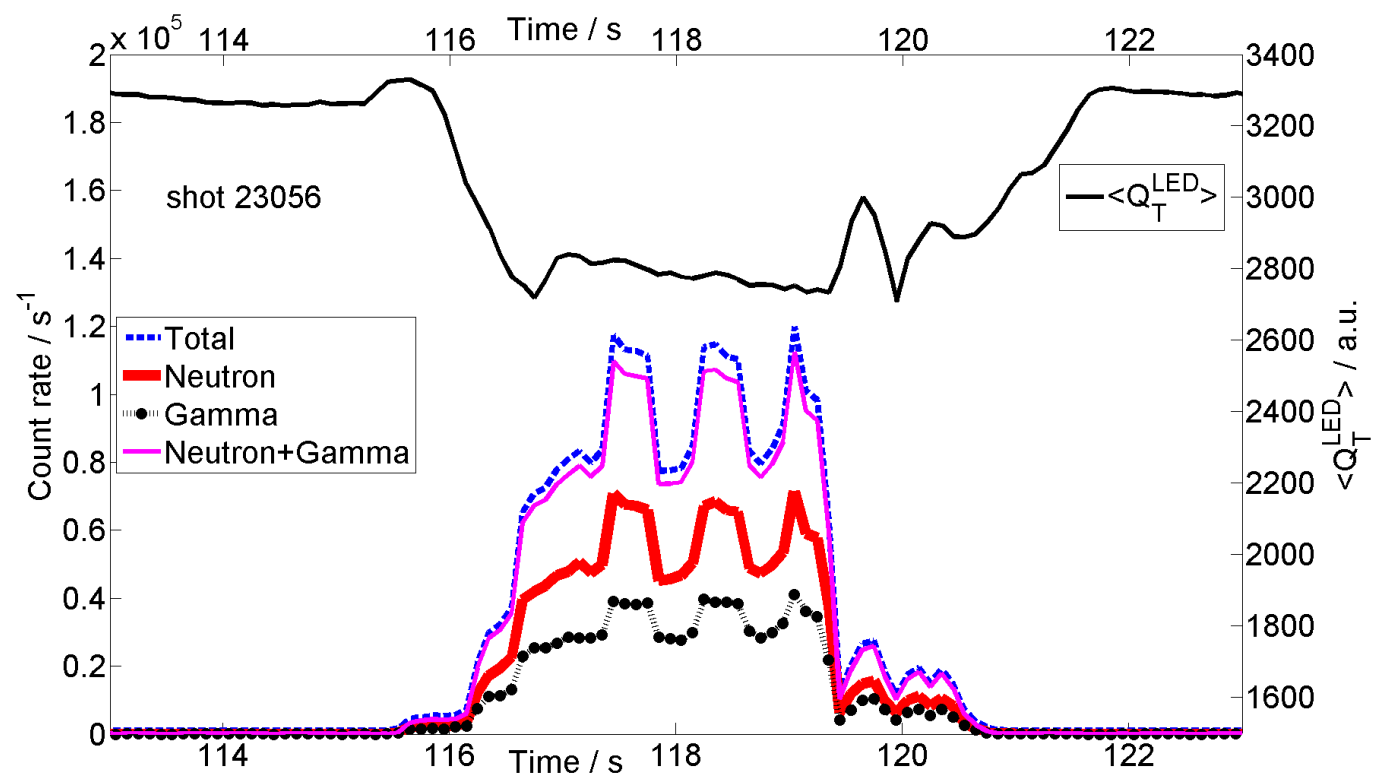

Fig. 3: (Color online) Count rates as a function of the DPSD acquisition time for the total, neutron and $\gamma$ events measured by the CNS in AUG discharge 23056. On the top, the evolution of the average LED pulse area $\left\langle Q_{T}^{L E D}>\right.$ during the discharge is shown. 


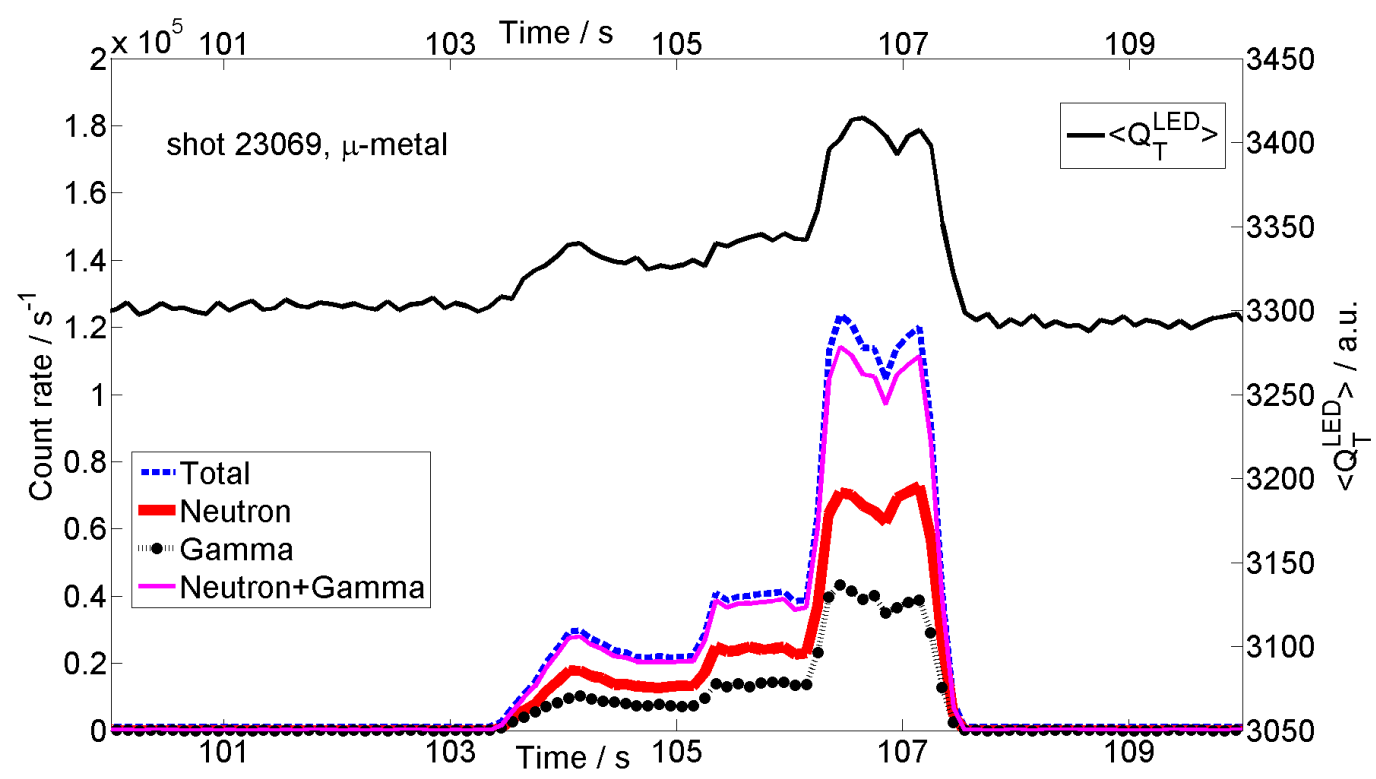

Fig. 4: (Color online) Same as in Fig. 3 but for AUG discharge 23069. The average LED pulse area $\left\langle Q_{T}^{L E D}>\right.$ follows exactly the count rate variation since the magnetic effects on the PMT are suppressed by the external $\mu$-metal shielding. 


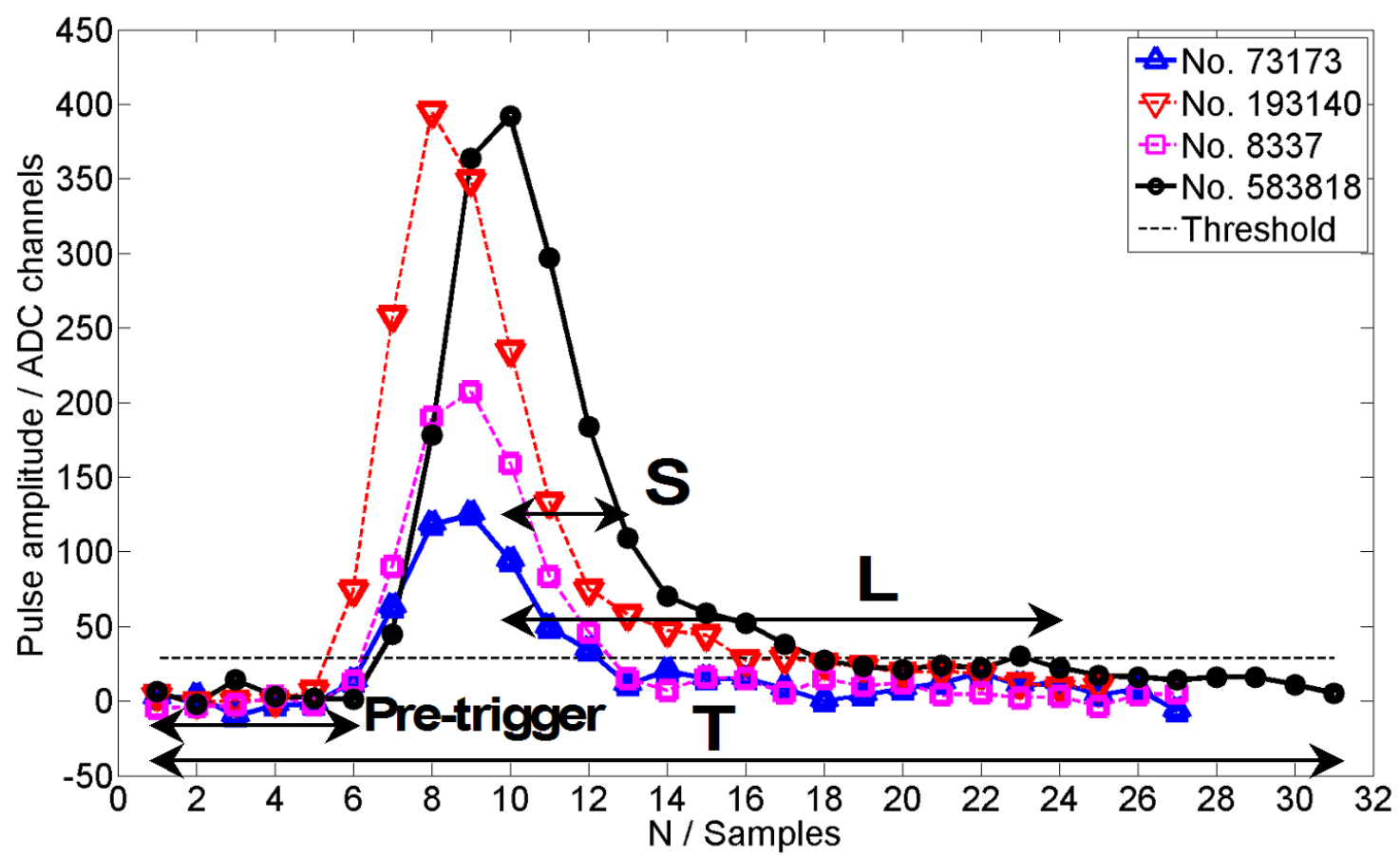

Fig. 5: (Color online) Examples of pulse shapes recorded in discharge 24151 with their corresponding number as recorded in the data set. Information is given on the DPSD acquisition trigger threshold at $10 \mathrm{mV}$. For pulse 583818, the regions of interests for $\mathrm{n}-\gamma$ discrimination, namely, pre-trigger and gates $\mathrm{S}, \mathrm{L}$ and $\mathrm{T}$ (i.e., the pulse length) are shown 


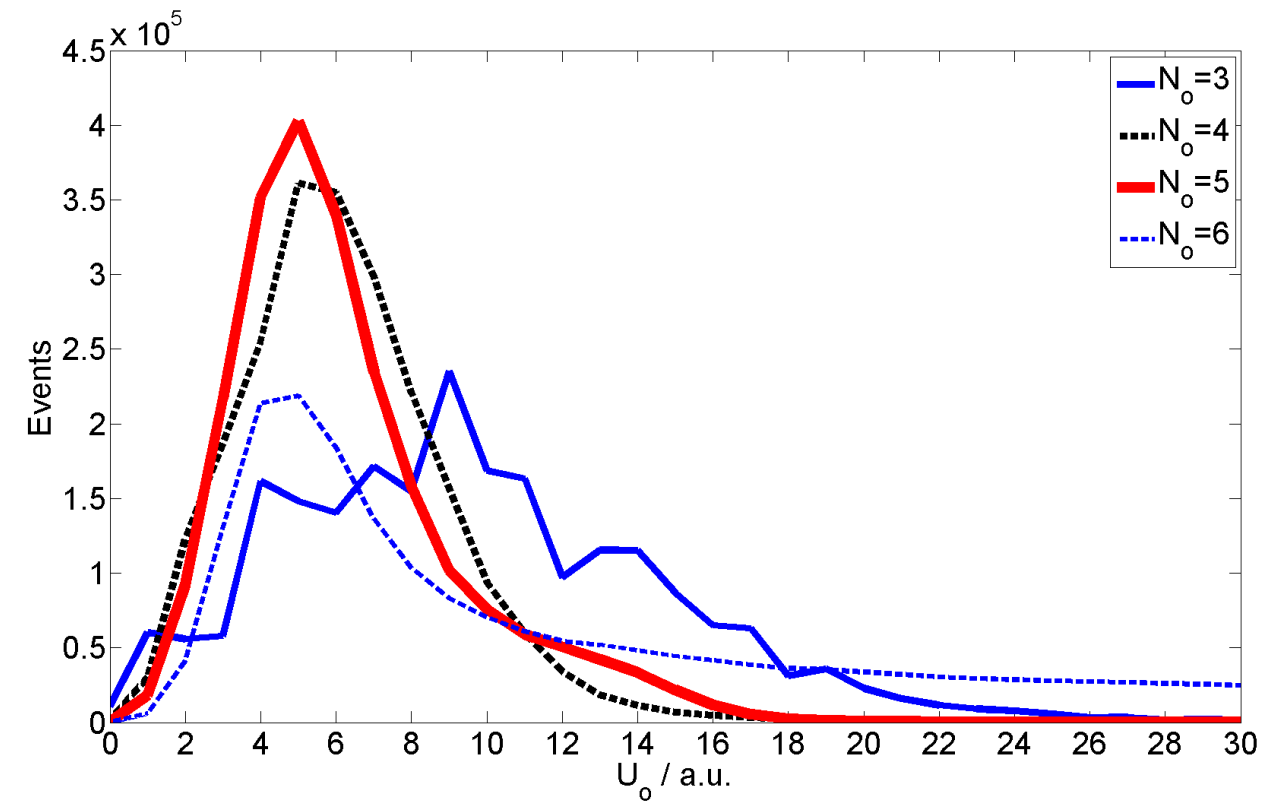

Fig. 6: Frequency distributions of the uncertainties $U_{0}$ associated to the offset value calculated considering a number of pre-trigger samples $\mathrm{N}_{\mathrm{o}}=[3,6] . \mathrm{N}_{\mathrm{o}} *=5$ samples is the optimal number of samples for the offset reduction of the data because of its lowest average uncertainty value of 5 a.u.. 


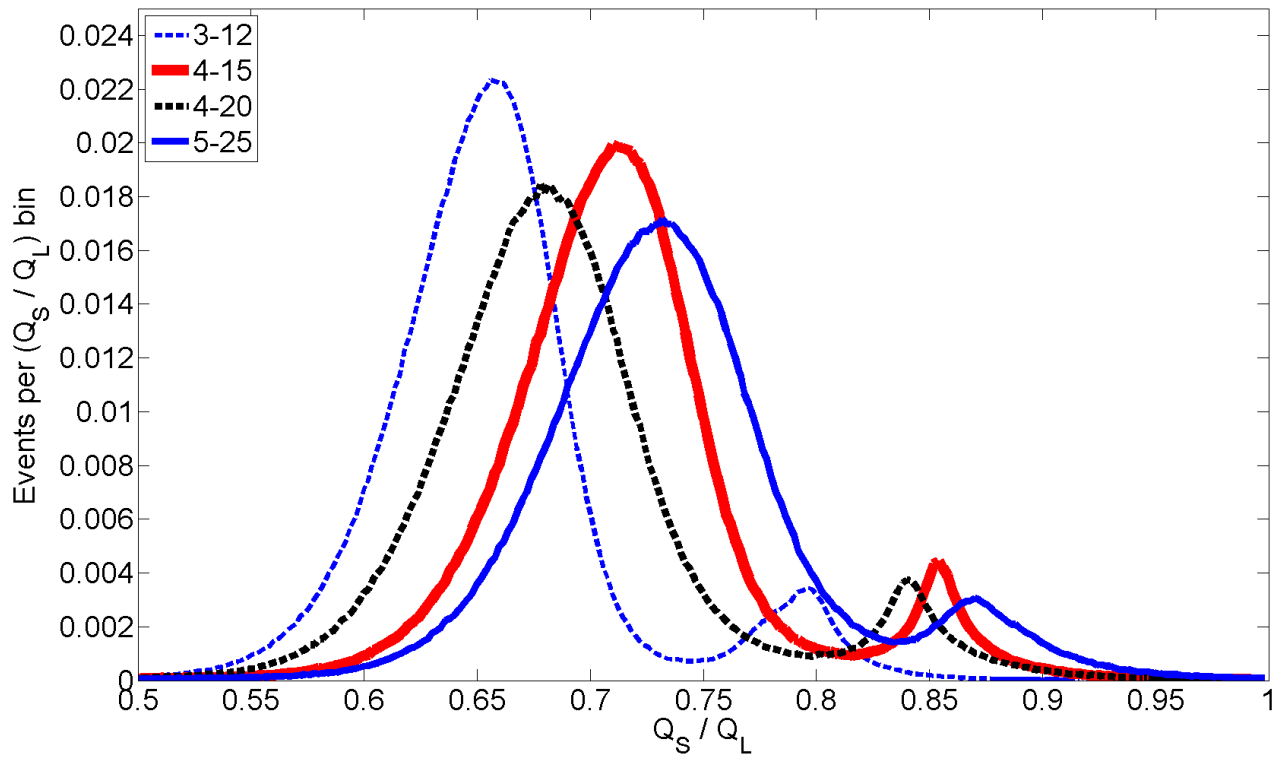

Fig. 7: (Color online) Examples of normalized $Q_{\mathrm{S}} / Q_{\mathrm{L}}$ distributions relative to different $\mathrm{S}$ L gate combinations. The peak on the left hand side corresponds to neutron events while the one on the right to $\gamma$ 's. The maximum FOM value of 1.38 corresponds to $S=4$ samples and $\mathrm{L}=15$ samples. 


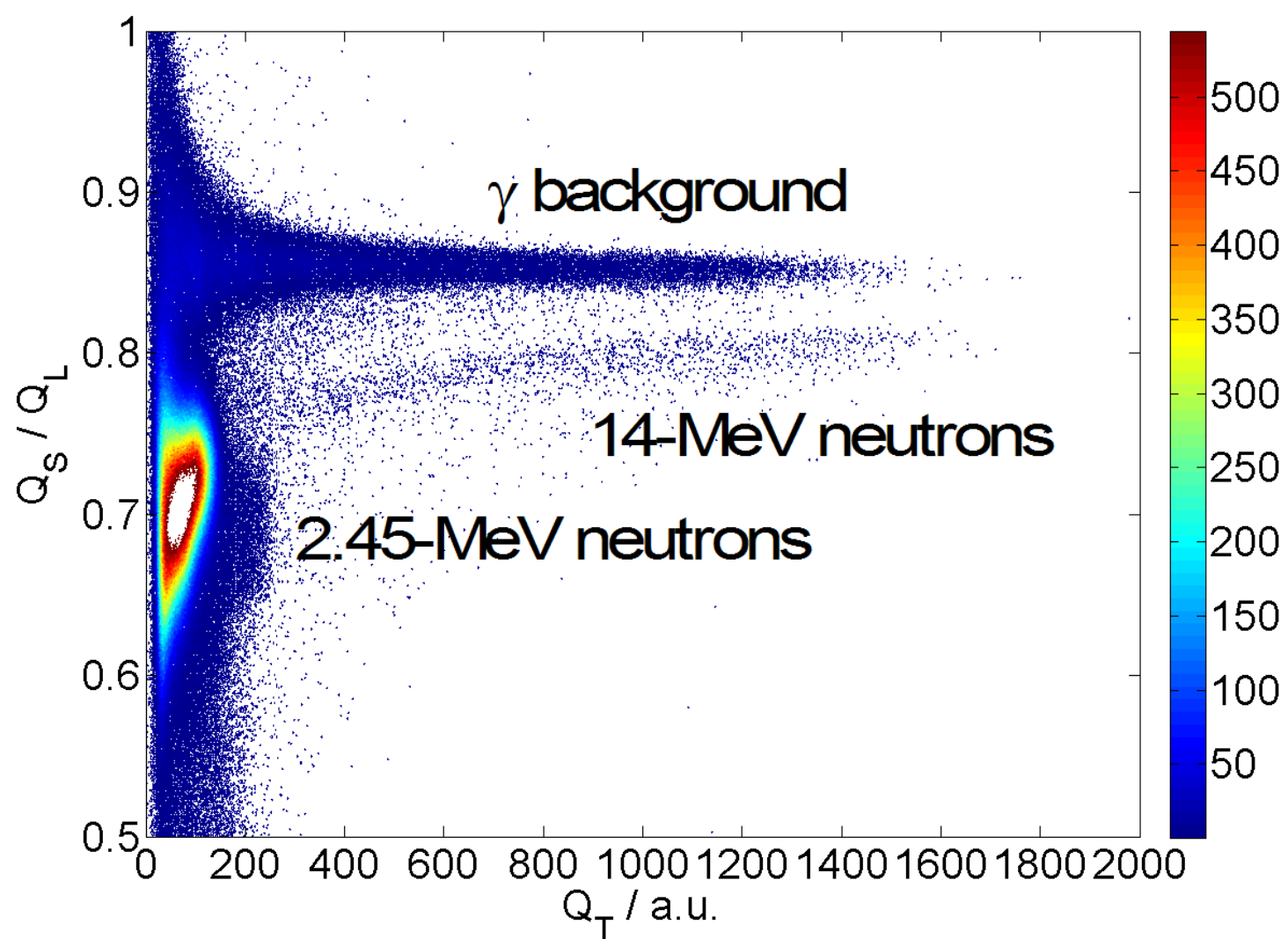

Fig. 8: (Color online) Contour plot of the radiation events recorded in AUG discharge 24151. The pulse shapes were offset reduced and LED corrected and analyzed using the optimal gates $\mathrm{S}=4$ samples and $\mathrm{L}=15$ samples. 


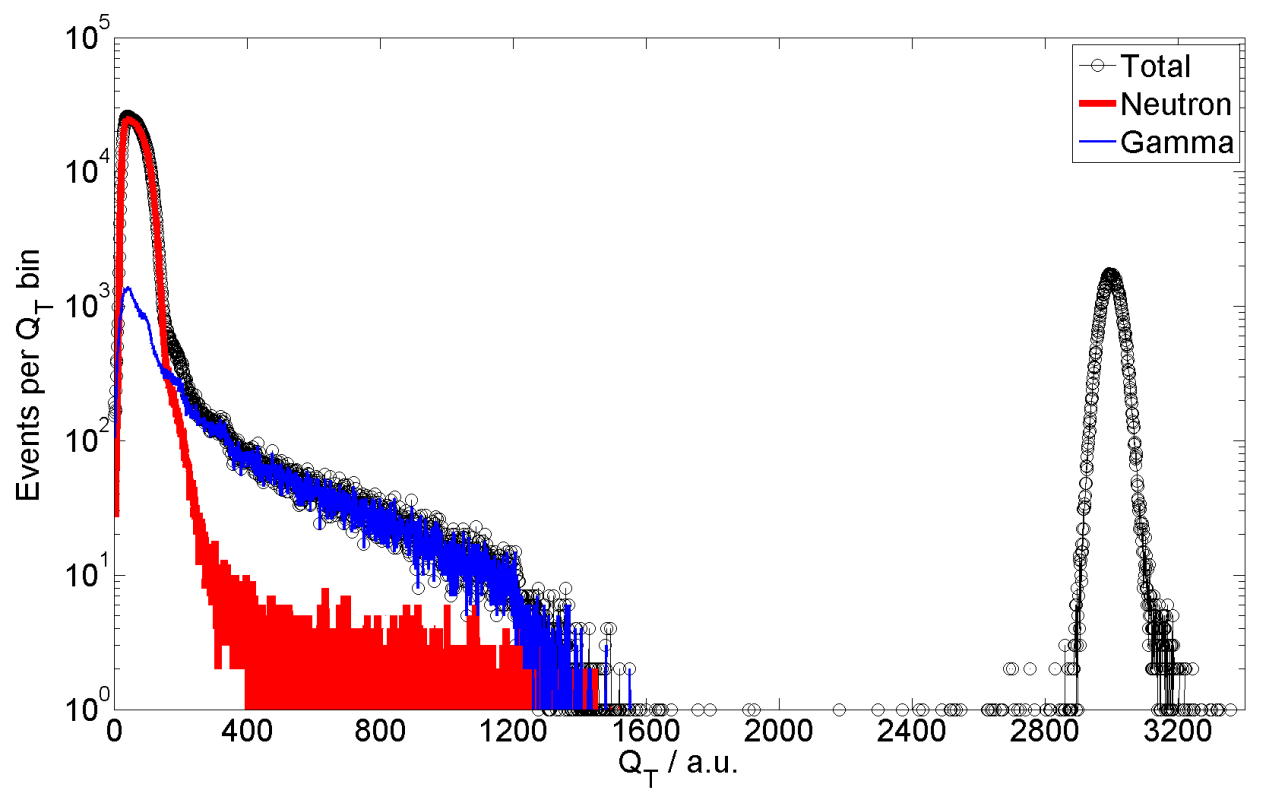

Fig. 9: (Color online) PHS $Q_{\mathrm{T}}$ of total, neutron, $\gamma$ and LED events. The LED events are peaked about the reference LED pulse area $Q_{\mathrm{T}}^{{ }_{\mathrm{LED}}^{*}}=3000$ a.u. after the PHS has been corrected. 


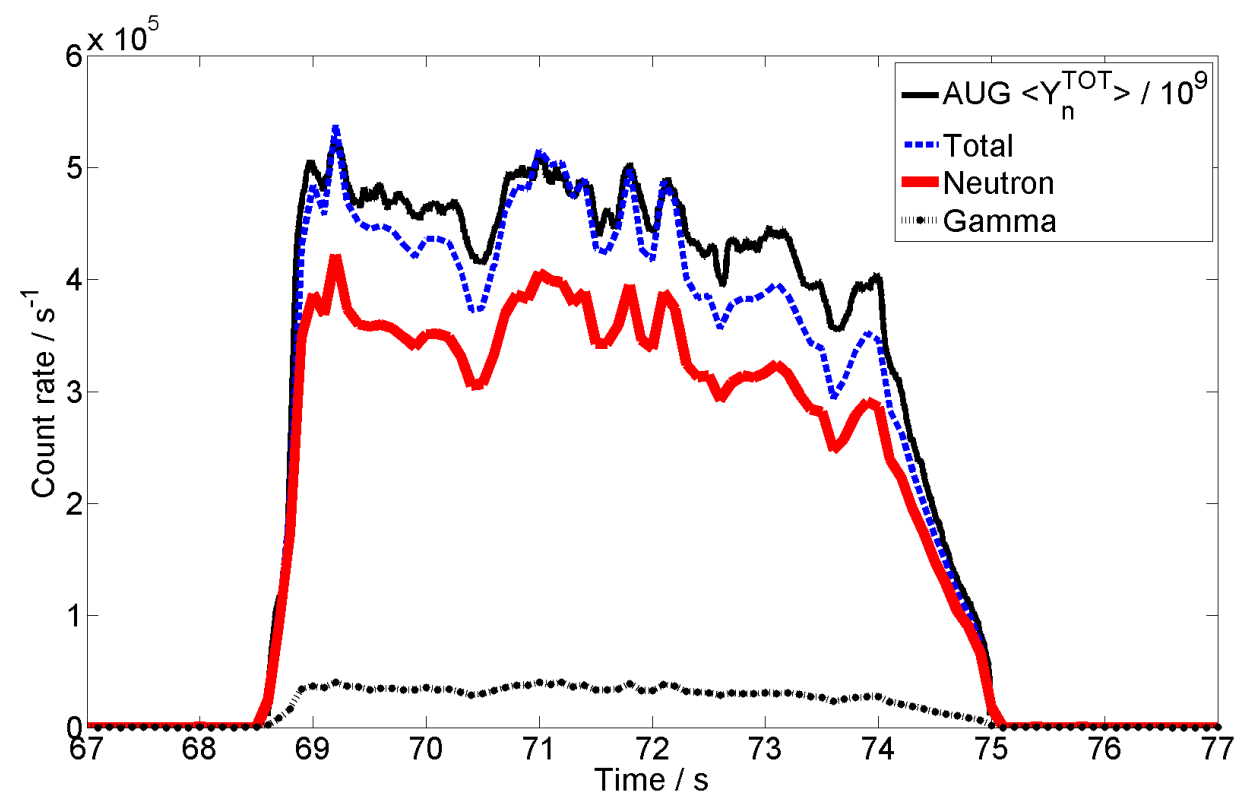

Fig. 10: (Color online) Comparison of count rates measured by the CNS with respect to the $100 \mathrm{~ms}$ time averaged AUG neutron yield $\left\langle\mathrm{Y}_{\mathrm{n}}{ }^{\mathrm{TOT}}>\right.$ (divided by $10^{9}$ ) during AUG discharge 24151. 